\title{
Study on the influence of standoff distance on substrate damage under an abrasive water jet process by molecular dynamics simulation
}

\author{
Ruling CHEN ${ }^{1, \S, *}$, Di ZHANG ${ }^{1,2, \S}$, Yihua WU ${ }^{2}$ \\ ${ }^{1}$ College of Mechanical Engineering, Donghua University, Shanghai 201620, China \\ ${ }^{2}$ Nano-Science and Technology Research Center, Shanghai University, Shanghai 200444, China \\ $\S_{\text {These authors contributed equally to this paper }}$ \\ Received: 09 February 2017 / Revised: 09 May 2017 / Accepted: 22 May 2017 \\ (C) The author(s) 2017. This article is published with open access at Springerlink.com
}

\begin{abstract}
The process of a cluster-containing water jet impinging on a monocrystalline silicon substrate was studied by molecular dynamics simulation. The results show that as the standoff distance increases, the jet will gradually diverge. As a result, the solidified water film between the cluster and the substrate becomes "thicker" and "looser". The "thicker" and "looser" water film will then consume more input energy to achieve complete solidification, resulting in the stress region and the high-pressure region of the silicon substrate under small standoff distances to be significantly larger than those under large standoff distances. Therefore, the degree of damage sustained by the substrate will first experience a small change and then decrease quickly as the standoff distance increases. In summary, the occurrence and maintenance of complete solidification of the confined water film between the cluster and the substrate plays a decisive role in the level of damage formation on the silicon substrate. These findings are helpful for exploring the mechanism of an abrasive water jet.
\end{abstract}

Keywords: standoff distance; crystalline silicon substrate; abrasive water jet; molecular dynamics simulation

\section{Introduction}

Abrasive water jet (AWJ) operation is described as the impact or collision process of the mixture of the water column and abrasives or clusters. AWJs are widely used in the machining filed, in processes such as abrasive water jet cutting, water jet cleaning, and abrasive water jet polishing (AWJP) [1-3]. Since being developed by Fähnle et al. in 1998 [3], AWJP was proposed to have a wide range of adaptability for machining surfaces, such as spheres, aspheres, and other free-form surfaces $[4,5]$, in order to obtain a nano-scale smooth surface.

It is widely understood that standoff distance is one of the most important operating parameters of AWJ processes. Generally, the depth of cut will almost linearly decrease as the standoff distance increases during AWJ operation [6, 7]. In addition, the optimum standoff will occur for material-removal processes, or surface damage of impacted substrates [8,9].

Based on the experimental phenomena mentioned above, the studies on the influence of standoff distance on substrate damage were conducted by Kordonski et al. They found that because of a combination of the absence of lateral limit by nozzle, surface tension variances, and aerodynamic disturbances, the degree of jet divergence increases progressively with the standoff distance [10]. This can make the energy density decrease and eventually lead to a lower jet penetration depth [11]. Thus, magnetorheological jet polishing (MJP), which was developed on basis of AWJ to realize the inhibition of jet divergence,

* Corresponding author: Ruling CHEN, E-mail: chen_ruling@163.com 
could preserve the stability of the material removal process, even if the standoff distance changes obviously [12].

Using computational fluid dynamics (CFD) simulation, Liu et al. verified that the velocities of the jet would rapidly decay as the standoff distance increases at the "initial region" during an AWJ process [13]. The low velocity will lead to a low degree of substrate damage [14]. In addition, Anglani et al. determined through CFD analysis that the shear stress on the impacted flat surface significantly increases as the standoff distance decreases [8]. In addition, He et al. showed that there is an optimum standoff distance for which the impact pressure reaches a maximum value [9] that is proportional to the substrate damage [14].

Thus far, most studies that focus on the effect of standoff distance on damage formation are performed based on a macroscopic view. However, as a result of evolving technology, the final machining accuracy for jet machining or polishing has become achievable at a nano-scale. And the details of AWJ machining process are still difficult to obtain through experimentation or CFD simulations, especially at nanometer or atomic scales. Therefore, there is a gap in knowledge between macroscopic phenomena and nano-mechanism regarding the effect of standoff distance on damage formation. At present, molecular dynamics simulation (MD or MDS) has been applied to investigate impact processes and solid-liquid two-phase flows. Therefore, it is possible to provide a feasible scheme to close this gap.

Over the past three decades, extensive molecular dynamics simulations have been conducted to study the damage of substrates under the impact of clusters. The "piling-up" shock phenomenon that occurs during the process of Ar cluster impacting with a sodium chloride surface was investigated by Cleveland and Landman [15]. The relationship between the depth of the crater and the incident energy of the cluster was also studied by some researchers through MD simulation, based on the ion bombardment process [16-18]. Aoki and Matsuo performed simulations of Ar cluster impacting on sine-curved $\mathrm{Si}$ surfaces and found that the impact process of the cluster varied depending on the surface structure around the impact point [19]. Han and Gan studied the surface planarization of the rough silicon surface under the impact of multiple nanoparticles [20]. Chen et al. studied the surface damage of the silicon surface covered by a water film [21] or silicon dioxide film [22] under silica cluster by MD simulations.

With respect to the process of solid-liquid two-phase flows, the impact and friction model [23] and the slip phenomenon [24] of $\mathrm{Ar}-\mathrm{Cu}$ mixed-phase nanofluid between two $\mathrm{Cu}$ plates were investigated through MD simulation by Lv and Aminfar, respectively. In addition, Algara-Siller et al. studied the structural properties of water in confined spaces using MD [25]. Bourg and Steefel studied the solidification phenomenon and diffusion process of confined water by performing an MD simulation of water-filled silica nanopores [26]. These studies are helpful for studying the physical properties of water beneath an abrasive in the AWJ process.

In summary, most of the simulation reports on cluster-surface interactions were performed without the use of a water jet (namely the dry impact individually of the cluster). Meanwhile, few simulations were performed using solid-liquid two-phase flows under the conditions of a water jet. A molecular dynamics study of the collision process of an abrasive water jet on a substrate is supposed to provide an atomistic insight into the dynamics of AWJ processes. However, detailed reports on the atomic-scale mechanism that explain the influence of standoff distance on the damage of the substrate during AWJ process are very few in number.

In this study, the mechanism of the collision process between the silicon substrate and the water jet, which contains an amorphous silica cluster under different standoff distances, was studied by molecular dynamics simulation at the nano-scale. In addition, the influence of standoff distance on the damage of the silicon substrate would be analyzed through the variance of a five-fold coordination number of silicon atoms.

\section{Simulation methodology}

\subsection{Simulation model}

Figure 1 illustrates the simulation model of the impact of a jet, which is composed of a water column and a 


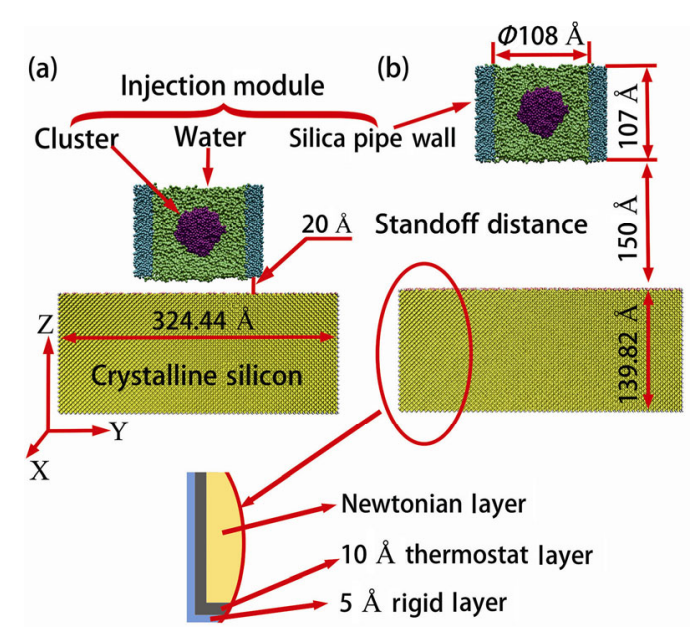

Fig. 1 Simulation model of an AWJ. (a) Standoff distance $=20 \AA$; (b) standoff distance $=150 \AA$.

cluster, toward a crystal silicon (c-Si) substrate. The $\mathrm{Si}$ (001) substrate contains approximately 499,200 atoms within a space of $215.84 \AA \times 324.44 \AA \times 139.82 \AA$. The outer layers ( $\sim 5 \AA$ thickness) of the substrate were fixed in space with the exception of the top contact surface. In addition, the thermostat layer $(\sim 10 \AA$ thickness) was used to simulate the heat conduction in a reasonable way.

The injection module was composed of a silica cluster, a silica pipe wall, and a water column with a cavity. Firstly, the amorphous silica cluster and silica bulk were prepared by quenching melted betacristobalite from $5,000 \mathrm{~K}$ and $7,000 \mathrm{~K}$ to $293 \mathrm{~K}$ at a cooling rate of $10^{14} \mathrm{~K} / \mathrm{s}$, respectively [27]. The silica cluster consists of 5,184 atoms with a diameter of approximately $54 \AA$. Secondly, the atoms of a pipe diameter of $108 \AA$ were removed from the center of the amorphous silica bulk, and then the amorphous silica pipe wall was prepared. The cluster surface and the inner surface of the silica pipe wall were composed of silicon atoms, bridging oxygen (BO) atoms bonded to two silicon atoms, and non-bridging oxygen (NBO) atoms bonded to one silicon atom. All NBO atoms were saturated with hydrogen atoms [28]. Thirdly, the silica cluster was placed in the cavity of the water column, whose diameter and length are $108 \AA$ and $107 \AA$, respectively. Finally, the jet was placed into the pipe of the silica wall.

The interatomic interaction among the silica cluster and the silicon substrate system was modeled by a Stillinger-Weber-like potential [29], and the cutoff distance of the interaction was $4.0 \AA$. The water was described by the TIP4P model [30]. The reaction field method was applied for the coulomb interactions between water molecules with a cutoff radius of $7.9 \AA$. The interactions between water and silicon substrate were described by an empirical Lennard-Jones potential model. The potential parameters were used and tabulated in Ref. [31], which provide further technical details. The cutoff distance for this Lennard-Jones interaction was $8.47 \AA$. Water molecules interacted with the silica atoms of the cluster and the pipe wall using the potential modeled according to Ref. [28], which consisted of Lennard-Jones and Coulomb interactions. And the pair interactions were truncated at a cutoff radius of $9 \AA$.

At the beginning of the simulation, the injection module was located at a standoff distance above the substrate surface. The simulation system was initiated with a temperature of $293 \mathrm{~K}$. After a relaxation of $7,000 \mathrm{fs}$ and a time step of $1 \mathrm{fs}$, the jet will impact the silicon substrate from the silica pipe with an initial impact velocity of $4,313 \mathrm{~m} / \mathrm{s}$ under different standoff distances. Therefore, the impinging kinetic energy of the cluster under the simulation condition is almost same as that under the AWJ experimental condition [32]. In this study, the impact processes of the jet with different standoff distances of $20 \AA, 30 \AA, 40 \AA, 50 \AA$, $75 \AA, 100 \AA, 125 \AA$ and $150 \AA$ were named Pos20, Pos30, Pos40, Pos50, Pos75, Pos100, Pos125 and Pos150, respectively. In order to study damage formation on the silicon substrate, the duration of the impact of the jet lasted for 8,000-15,000 fs. The larger the standoff distance, the longer the duration time. During the entire impact process, the atoms of the silica pipe wall were fixed. The temperature of the thermostat atoms of the silicon substrate was kept at $293 \mathrm{~K}$ by using Gaussian constraint thermostat method [33].

\subsection{The calculation of local temperature and pressure}

To calculate the local pressure and temperature of the ensemble, the section of the x-direction from $102 \AA$ (Xmin) to $114 \AA$ (Xmax) was chosen, as shown in Fig. 2 . The silicon substrate and water film were divided by the grid of $12 \AA \times 12 \AA \times 12 \AA$ and $12 \AA \times 5 \AA \times 3.5 \AA$, respectively. In order to reduce the statistical error, each grid is partially overlapped, as shown in Fig. 2(a). 


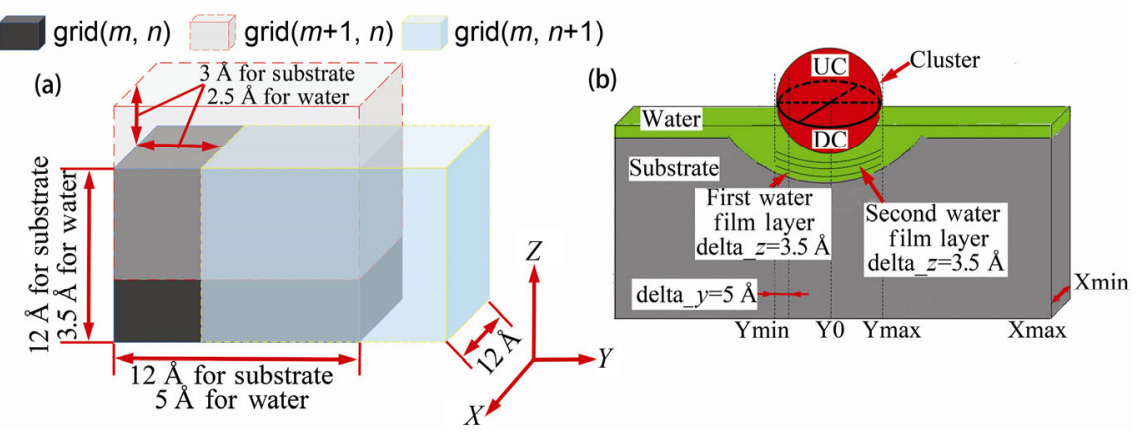

Fig. 2 (a) Calculation mode of local temperature and pressure of substrate or water film. (b) Calculation mode of density of water film layer and cluster force.

For the silicon substrate and the water film, each grid is separated in the $y$ or $z$ direction by $3 \AA$ and $2.5 \AA$, respectively.

\subsection{The calculation of water film layer density}

In order to further study the variation in the density of the water film, the water on the substrate surface in the section is divided into several layers, as shown in Fig. 2(b). The $y$ direction of each layer ranges from 135-190 A. Starting from the silicon substrate surface, every delta_z $z=3.5 \AA$ is referred to as a layer. Then, each layer was divided into a number of grids by delta $\_y=5 \AA$ in the $y$-direction. Finally, the water density of each layer is obtained by averaging the water density of all grids in this layer.

\subsection{The calculation of cluster force}

The force between the cluster and the silicon substrate is defined as $\overrightarrow{F_{\mathrm{C}-\mathrm{S}}}$. In addition, the cluster was further divided into two hemispheres by the plane that passes through the cluster centroid and is parallel to the XOY plane, denoted as upper-cluster (UC) and down-cluster (DC), as shown in Fig. 2(b). Then, $\overrightarrow{F_{\text {UC-W }}}$ and $\overrightarrow{F_{\mathrm{DC}-\mathrm{W}}}$ represent the force from the water molecules on the UC and DC atoms, respectively. Obviously, a resultant force on the cluster $F$ is the sum of $\overrightarrow{F_{\mathrm{C}-\mathrm{S}}}$, $\overrightarrow{F_{\mathrm{UC}-\mathrm{W}}}$ and $\overrightarrow{F_{\mathrm{DC}-\mathrm{W}}}$. In this study, the forces in the $z$-direction are our focus points.

\section{Simulation results}

As shown in Fig. 3, the deformation area will appear near the impact zone when the abrasive water jet impinged on the substrate. Meanwhile, the crystalline silicon will begin to amorphize in the deformation area due to large temperature and pressure gradients. That means that the single-crystal silicon atoms with a four-fold coordination number (CN4 atom) were converted into the atoms with three- and five-fold coordination number, respectively. They were referred to as the $\mathrm{CN} 3$ and $\mathrm{CN} 5$ atoms, respectively. According to previous studies [21,34], it was found that the volume of the damaged region corresponds to the volume of the deformation region when the number of $\mathrm{CN} 5$ atoms reaches a maximum. Therefore, the variance in the number of $\mathrm{CN} 5$ atoms is selected as a quantitative criterion for judging the degree of damage incurred by the crystalline silicon. The higher the maxima of the number of $\mathrm{CN} 5$ atom (CN5max), the larger damage region.

Figure 4 shows that $\mathrm{CN} 5 \mathrm{max}$ varies inconspicuously when the standoff distance ranges from 20-40 A. When the standoff distance is more than $40 \AA$, CN5max decreases rapidly as the standoff distance increases. This is consistent with the variance in the damage severity of the substrate according to Fig. 3.

The above simulation shows that there is a critical value of the standoff distance (about $40 \AA$ ). When the standoff distance does not exceed the critical value, the substrate damage will inconspicuously change. Once the critical value is exceeded, the substrate damage will rapidly decrease. This phenomenon is consistent with the experimental conclusion that an optimal standoff distance exists in the AWJ experiment [8]. 

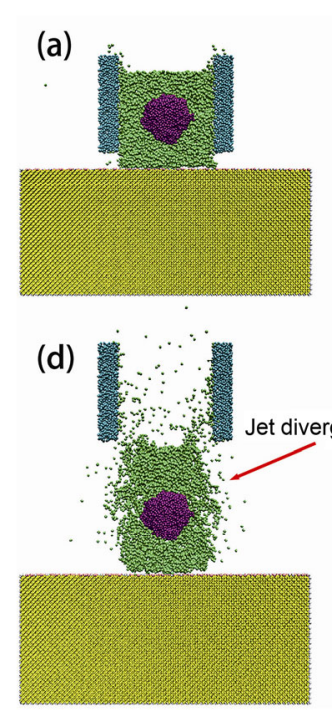

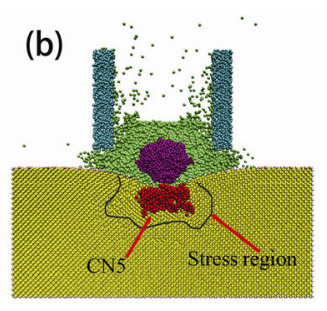

(e)

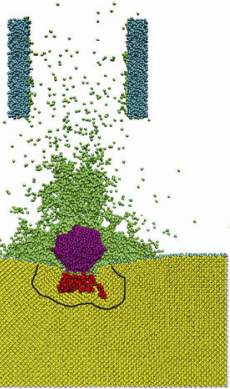

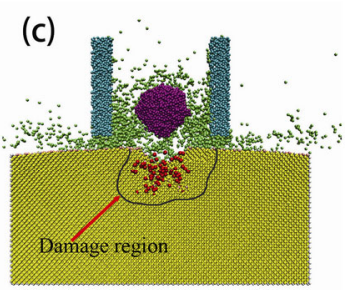

(f)

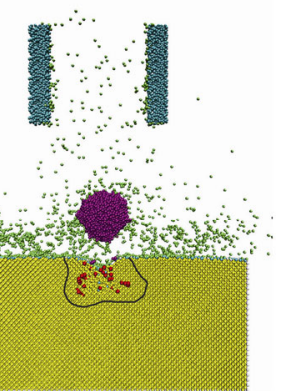

Fig. 3 Side cross-section view of the impact zone at different moments. Red particles represent CN5 atoms. (a), (b), (c) are the snapshots of $750 \mathrm{fs}, 1,950 \mathrm{fs}$, and 6,000 fs at Pos20, respectively. (d), (e), (f) are the snapshots of 3,750 fs, 4,900 fs, and 8,000 fs at Pos150, respectively. (a) and (d) represent the moment when the jet first contacts the substrate; (b) and (e) represent the moment of the maximum of the number of $\mathrm{CN} 5$ atom; (c) and (f) represent the end of the impact process.
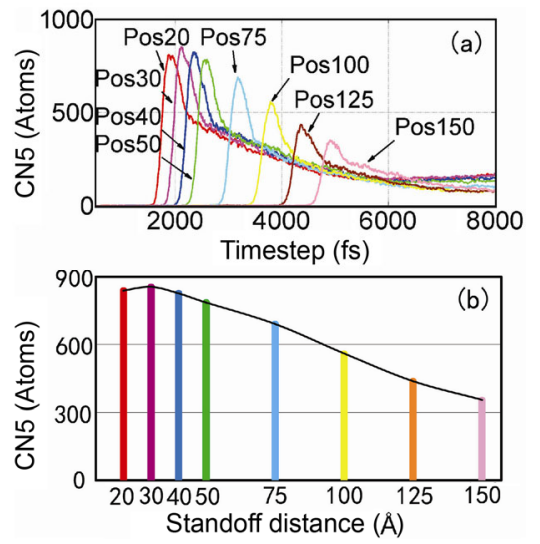

Fig. 4 (a) The CN5 variation curve with time during jet impingement on substrate with different standoff distances. In order to make figures clear, the data after 8,000 fs was not shown; (b) the CN5max variation curve with different standoff distances.

\section{Analyses and discussions}

The relationship between the standoff distance and the substrate damage was studied by analyzing the variance of $\mathrm{CN} 5$ number as follows. The jet process was divided into four stages.

\subsection{The stage of solidification formation of the water film (Stage_I)}

At the beginning of the simulation, the jet moves from the nozzle to the substrate at an initial velocity of $4,313 \mathrm{~m} / \mathrm{s}$. Then, the jet front will contact with the substrate first.

When the jet front reaches the substrate, it will form a water film on the substrate surface due to the impediment from substrate. With the impact proceeding, the density of this water film will exceed the value of density $\left(1.0 \mathrm{~g} / \mathrm{cm}^{3}\right)$ of water under the normal condition (Fig. 5), and continue to be higher. We refer to this process as "the water film solidifying phenomenon". At the same time, the cluster gradually approaches the solidified water film. Thus, the interaction between the cluster and the water film will begin to gradually appear (Fig. 6(a) at $750 \mathrm{fs,}$ Fig. $6(\mathrm{~b})$ at 3,750 fs). The period from the initial time of the simulation to the appearance of the interaction between the cluster and water film is called the stage of solidification formation of the water film (Stage_I).

On the other hand, when the water film is solidified, the local temperature and pressure of the substrate in the impact zone begin to increase as the impact energy of the jet increases (Fig. 7). Moreover, the impact energy of the substrate only stems from the action of the water because there is no interaction between the cluster and water film at this stage (Fig. 6).

For small standoff distances (from Pos20 to Pos40), 
the shape and velocity distribution of the jet front does not change much compared to the starting time at the moment when the jet front contacts the substrate. On the contrary, when the standoff distance is over $40 \AA$, the shape (Figs. 3(a) and 3(d)) and velocity distribution of the jet front (Fig. 8) will be different from the starting time because of the weakness of the restriction of the nozzle. This phenomenon is called jet divergence. Obviously, the larger the standoff distance, the more serious the jet divergence will be.
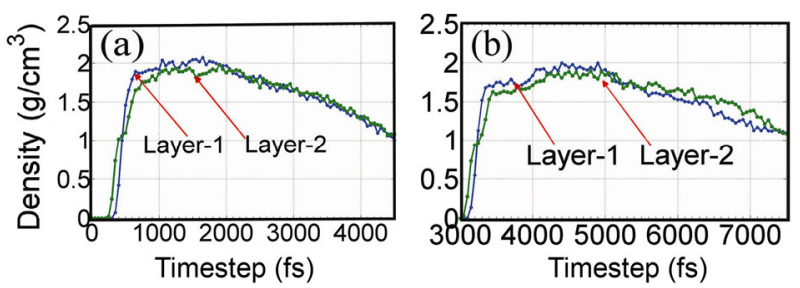

Fig. 5 The average density variation curve with time of the water film layer on the substrate surface: (a) Pos20; (b) Pos150.
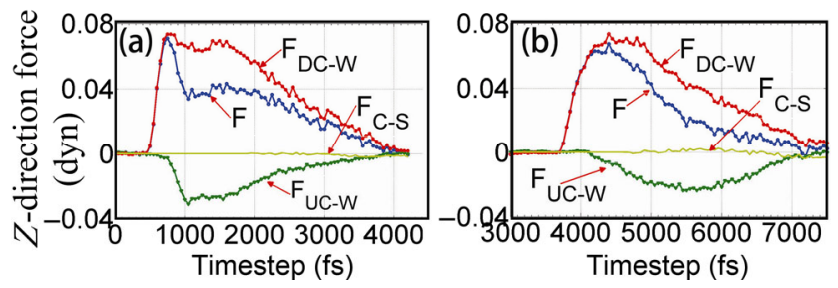

Fig. 6 Z-direction forces variation curve with time on cluster: (a) Pos20; (b) Pos150.

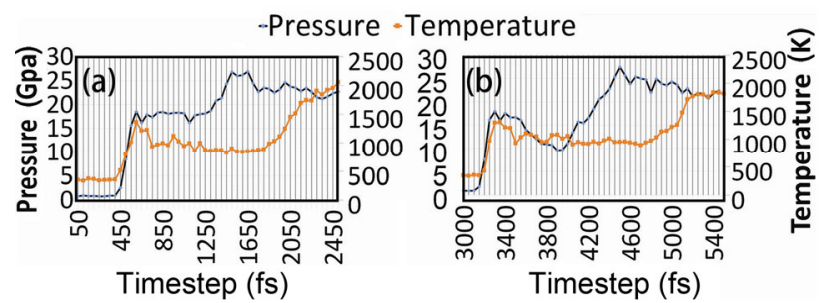

Fig. 7 The maximum pressure variation curve of the impact zone of the silicon substrate: (a) Pos20; (b) Pos150.
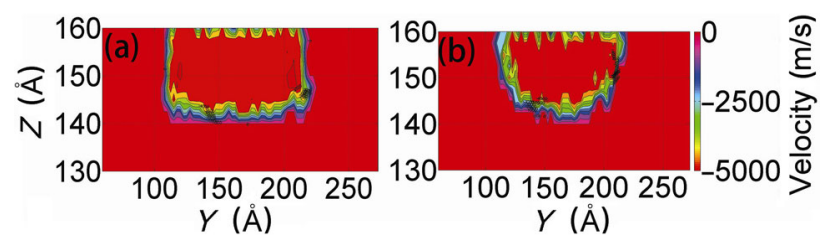

Fig. 8 The velocity distribution of the water in the $\mathrm{z}$-axis direction when the jet first contacts with the substrate. (a) Pos20 at $750 \mathrm{fs}$; (b) Pos 150 at 3,750 fs.
On the one hand, jet divergence has no effect on the velocity of the cluster, but will elongate the thickness of the water film, which is defined as the distance between the cluster and the silicon substrate. As shown in Fig. 9, this is not favorable to water film solidification. Therefore, the density of the solidified water film at Pos150 $\left(1.6-1.75 \mathrm{~g} / \mathrm{cm}^{3}\right)$ is significantly lower than that at Pos20 $\left(1.75-1.9 \mathrm{~g} / \mathrm{cm}^{3}\right)$ at the end of this stage. Meanwhile, the thickness of the solidifying water film (20 ̊) in Pos150 is larger than Pos20 (17 $)$ ), as shown in Figs. 9-11. In other words, the water film of Pos150, is relatively "loose" and "thick", compared to Pos20. This will result in a decrease in the energy transfer efficiency of the solidified water film, which is not favorable to the increase in the local pressure and temperature of the substrate due to the decreasing of input energy absorbed by the substrate.

On the other hand, because of the jet divergence, the time required for the solidification of the water film is prolonged. This means that the local pressure of the impact zone has more time to diffuse in the substrate, which is also not favorable to the increase in local pressure.

Because of the two reasons mentioned above, the local pressure of the impact zone of the substrate at Pos150 will increase firstly and then decrease (Fig. 7). The stress region (whose pressure is over $9 \mathrm{GPa}$ on basis of the transformation pressure from Si-I to Si-II [35]) of Pos150 is larger than that of Pos20 at the end of the stage. However, the maximum pressure and high-pressure region (whose pressure is over $15 \mathrm{GPa}$ ) of the substrate at Pos150 are less than these at Pos20 (Figs. 12(a) and 13(a)). The high-pressure region was defined as the region of the local pressure over $15 \mathrm{Gpa}$ because the distribution area of CN5 is in good
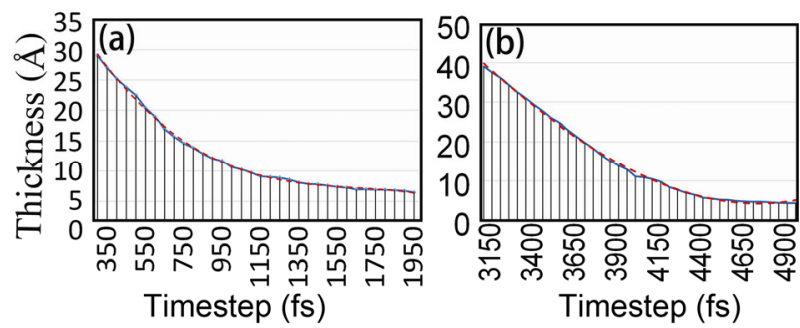

Fig. 9 The thickness variation curve with time of the water film confined between the cluster and the silicon substrate: (a) Pos20; (b) Pos 150 . 

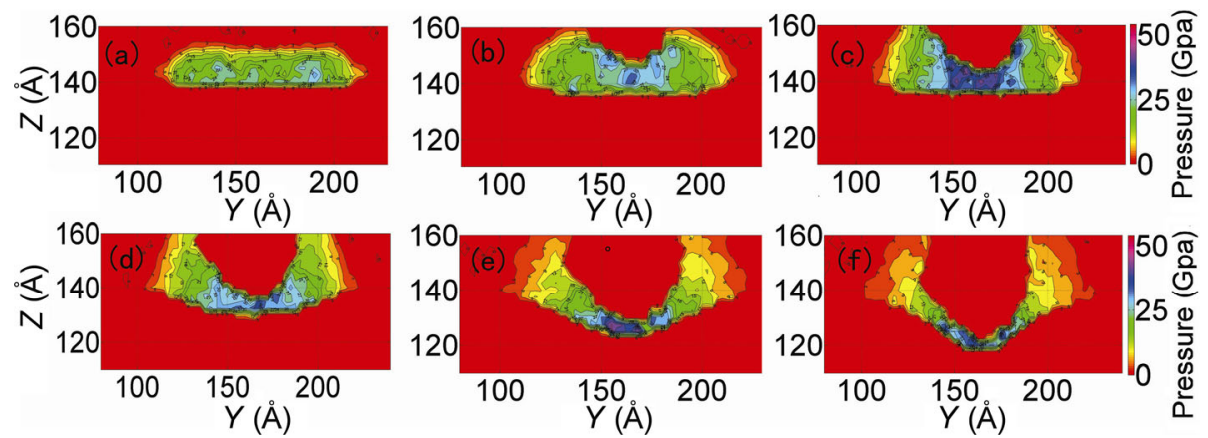

Fig. 10 Pressure distribution of water film confined at different moments under Pos20. Stage_I, Stage_II, and Stage_III range from 0-750 fs, 750-1,050 fs, and 1,050-1,950 fs, respectively. (a) $750 \mathrm{fs}$; (b) $900 \mathrm{fs}$; (c) 1,050 fs; (d) 1,300 fs; (e) 1,600 fs; (f) $1,950 \mathrm{fs}$.
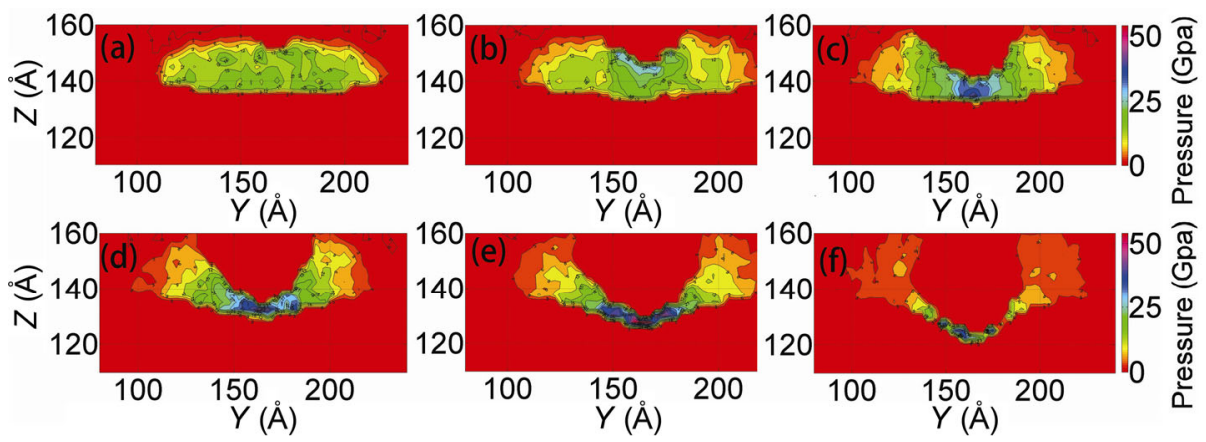

Fig. 11 Pressure distribution of water film confined at different moments under Pos150. Stage_I, Stage_II, and Stage_III range from 0-3,750 fs, 3,750-4,100 fs, and 4,100-4,900 fs, respectively. (a) 3,750 fs; (b) 3,900 fs; (c) 4,100 fs; (d) 4,300 fs; (e) 4,500 fs; (f) 4,900 fs.
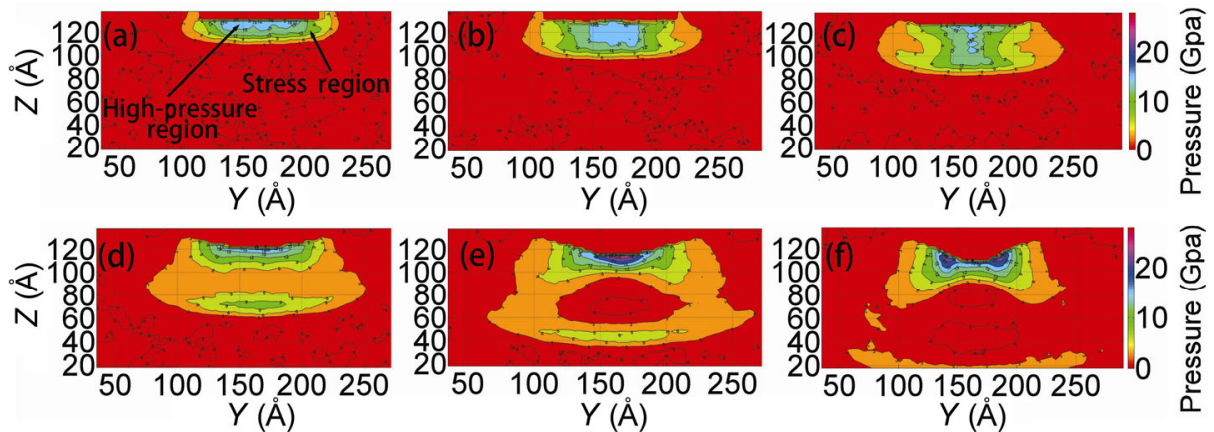

Fig. 12 Pressure distribution of silicon substrate at different moments under Pos20. (a) $750 \mathrm{fs}$; (b) $900 \mathrm{fs}$; (c) 1050 fs; (d) 1,300 fs; (e) $1,600 \mathrm{fs}$; (f) $1,950 \mathrm{fs}$.
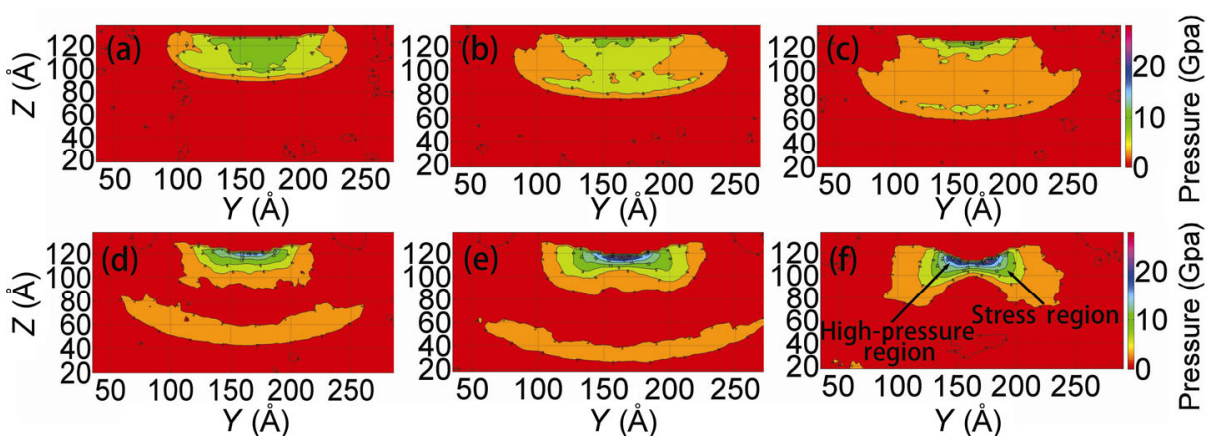

Fig. 13 Pressure distribution of silicon substrate at different moments under Pos150. (a) 3,750 fs; (b) 3,900 fs; (c) 4,100 fs; (d) 4,300 fs; (e) 4,500 fs; (f) 4,900 fs. 
agreement with that of over 15 GPa pressure regions at the moment of CN5max (Figs. 2(b), 3(b), 12(f), and 13(f)). In this stage, the CN5 atom was not noticed in the impact zone of the substrate, as shown in Fig. 4.

\subsection{The stage of complete solidification of the water film (Stage_II)}

When the cluster begins to contact the solidified water film and then compress it, the force of the cluster in the $z$-direction $\left(F_{z}\right)$ increases quickly from zero as the cluster moves downward (Fig. 6). Meanwhile, the thickness of solidified water film decreases (Fig. 9). Therefore, the density and the local pressure of the solidified water film increase further (Figs. 5, 10, and 11). When $F_{z}$ reaches its maximum $\left(F_{z \max }\right)$, the density and the local pressure reach their maximum values as well (Fig. 5), which means that the water film achieves a full degree of solidification. The period spreading from the appearance of $F$ to the moment of $F_{z \max }$ is called the stage of complete solidification of water film (Stage_II).

It is shown in Fig. 6 that there is no direct contact between the cluster and the substrate, and that the water film plays an intermediate role in the energy transfer from the cluster to the silicon substrate. That is to say, on the one hand, the water film solidification absorbs a part of the impact energy of the cluster; on the other hand, the remaining impact energy is transferred by the solidified water film to the silicon substrate. The high-pressure area of water film at Pos20 is evenly distributed in a larger hemispherical area below the cluster (Figs. 10(a)-10(c)) to incur the impact load of the jet. Therefore, the stress region at Pos20 at this stage rapidly increases because the impact energy of the cluster is evenly distributed over a larger area of the substrate. However, the highpressure region of the substrate is slightly decreased (Figs. 12(a)-12(c)).

In contrast with Pos20, the high-pressure area of the water film at Pos150 starts concentrating to a relatively small area just below the cluster, which is "thick" and "loose" (Figs. 11(a)-11(c)). Therefore, the majority of the input energy of the cluster is used to achieve complete solidification of the water film, and a very small part is transferred into the silicon substrate through the water film in this stage. Therefore, the maximum pressure and the stress region of the substrate reduce gradually. In addition, the high-pressure region diminishes or disappears (Figs. 13(a)-13(c)).

Therefore, the maximum pressure and the highpressure region of the silicon substrate in Pos20 continues to be larger than those in Pos150. Moreover, the stress region of the former is also significantly larger than that of the latter.

\subsection{The stage of maintaining complete solidification of the water film (Stage_III)}

The cluster continues to move down after the water film is completely solidified. The period from the moment of $F_{z \max }$ to the moment of $\mathrm{CN} 5 \mathrm{max}$ is called the stage of maintaining complete solidification of the water film (Stage_III), because the density of the solidification film experiences only a small change during this stage (Fig. 5).

At this stage, the input energy of the cluster and the water is mainly divided into two parts: (1) one part overcomes the resistance of the solidification water film in order to reduce the thickness of the solidification film (E1); (2) another part transfers into the silicon substrate by the solidifying water film (E2).

For the standoff distance at Pos20, E1 is larger than E2 (E1>E2) during the early period of this stage. Therefore, the area where the solidifying water film is sustaining the load of the jet is relatively large (Figs. 10(c) and 10(d)). In addition, the stress wave will split at the stress region of the substrate because E2 is not sufficient. Therefore, the stress region of the substrate will decrease significantly. However, the high-pressure region of the substrate gradually increases, and the maximum pressure of the substrate cannot increase (Figs. 12(c) and 12(d)).

With the continuous embedding of the cluster, the area where the solidifying water film is sustaining the load of the jet becomes more and more concentrated under the cluster (Figs. 10(d) and 10(e)). This causes E1 to gradually become equal to E2, even smaller than E2. Therefore, the stress region of the silicon substrate slowly increases again in the period of E1 $\leqslant$ E2 (as Figs. 12(d) and 12(e)), but the high-pressure region and the maximum pressure significantly increased.

For Pos150, the area where the solidifying water 
film sustains the load of the jet has concentrated under the cluster during the early period of this stage (Figs. 11(c)-11(e)). Therefore, it directly enters the period of $\mathrm{E} 1 \leqslant \mathrm{E} 2$. As a result, the local pressure of the stress region of substrate increases rapidly and the high-pressure region appears again and gradually increases (Figs. 13(c)-13(e)). However, the stress region and high-pressure region at Pos150 are still significantly lower than those at pos20 when the CN5 atom appears.

When the maximum pressure of the substrate exceeds the threshold value for the transition between the $\mathrm{CN} 4$ atom and the CN5 atom, CN5 atoms will appear and the cluster does not continue to be embedded in the substrate. As shown in Fig. 9, Pos20 and Pos150 are stable at approximately $7 \AA$ and $4.5-5 \AA$, respectively. This means that $\mathrm{E} 1$ is much smaller than E2 during this period $(\mathrm{E} 1<<\mathrm{E} 2)$. It is noticed that the maximum pressure of the substrate will slightly reduce first, and will then experience only a minor change whether the standoff distance is Pos20 or Pos150. In addition, the areas of the stress region and high-pressure region increase very slowly after CN5 appears (Figs. 12(e), 12(f), 13(e), and 13(f)).

Under continuous impact energy, the number of CN5 atoms rapidly increases to a maximum at 1,950 fs (Pos20) and 4,900 fs (Pos150), respectively. Therefore, the period from when the $\mathrm{CN} 5$ atom appears to the moment when the maximum number of $\mathrm{CN} 5$ atoms is obtained in Pos20 is as same as that in Pos 150 (Fig. 4(a)).

Meanwhile, there is no obvious difference in the local pressure and temperature maximum of the impact zone between Pos20 and Pos150 at the moment of CN5max.

Therefore, CN5max of Pos20 is significantly larger than that of Pos150 because the areas of the stress region and high-pressure region of the former are greater than those of the latter.

\subsection{The stage of degradation of the complete solidified water film (Stage_IV)}

As the velocities of the cluster and the water behind the cluster further decrease, the density of the solidifying water film begins to decrease (Fig. 5). This process is known as the degradation phenomenon of the solidified water film (Stage_IV). This will result in a decrease in the transfer-efficiency of the water film, which leads to a reduction in the energy absorbed by the silicon substrate. As a result, the local pressure of the substrate in the impact zone will decrease. In addition, the local temperature of the substrate will rise due to the gradual weakening of the restriction from the densifying water film. Therefore, the number of CN5 atom decreases rapidly (Fig. 4). Finally, the substrate enters a state of slow relaxation with the rebounding of the jet. The substrate damage gradually formed and held stable, along with the mutual transformation between $\mathrm{CN} 5, \mathrm{CN} 3$, and $\mathrm{CN} 4$.

\subsection{Discussions}

The formation of damage is in accordance with the variance in the number of CN5 atoms in the impact zone of the silicon substrate during the water jet process. The factors that influence the formation of the damage are listed as follows: (1) the stress and high-pressure regions at the moment of $\mathrm{CN} 5 \mathrm{max}$; (2) the maximum of local pressure and local temperature at the moment of $\mathrm{CN} 5 \mathrm{max}$; (3) the duration from the moment of the appearance of CN5 atom to the moment of CN5max. However, no significant difference was observed for the latter two factors under different standoff distances with the same impact velocity. Therefore, the influence of standoff distance on the degree of damage incurred by the substrate is mostly contributed by the first factor.

As shown in Fig. 14, the solidification of the jet front will occur under the impact of the water jet during the water film solidification stage. However, as the standoff distance increases, the water jet will diverge gradually. Therefore, the solidifying water film formed by a small distance is relatively "dense" and "thin", while the solidifying water film formed by large distance is relative "loose" and "thick". As a consequence, the jet front will spend more time solidifying at large distances, which results in a longer diffusion time for the stress region of the substrate. Therefore, at the end of this stage, the area of the stress region at small distance is smaller than that at large distance, but the area of the high-pressure region of the former is larger than that of the latter.

The cluster begins to compress the water film during 


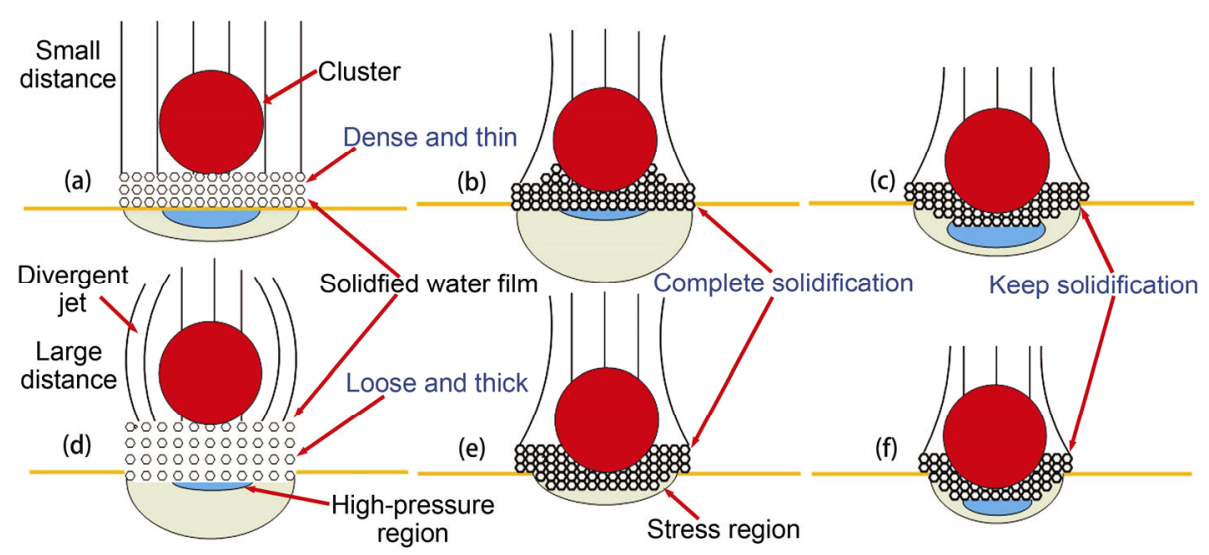

Fig. 14 Sketch of the process of AWJ impingement on substrate. (a), (b), and (c) are at small standoff distance; (d), (e), and (f) are at large standoff distance. (a), (d) represent the moment that the water film starts to solidity; (b), (e) represent the moment of the water film complete solidification; (c), (f) represent the moment that substrate reaches maximum damage.

the stage of complete solidification of the water film. Firstly, most of the input energy of the cluster is used for realizing the complete solidification of the water film. Secondly, the remains are transferred to the silicon substrate by the solidification film. As the standoff distance increases, the energy absorbed by the substrate becomes smaller and smaller. For small standoff distances, the impact energy of the cluster could be absorbed by a larger area of the substrate because the solidifying water film is denser. This can make the stress region increase rapidly, but is not enough to maintain the high-pressure region. Therefore, the high-pressure region decreases. For large standoff distances, both stress region and high-pressure region reduced significantly. And the high-pressure will even disappear. Therefore, at the end of this stage, both the area of stress region and high-pressure region at small distances are significantly larger than those at large distances.

At the stage of maintaining complete solidification of water film, the relationship between E1 and E2 usually goes through three periods: E1 $>$ E2, E1 $\leqslant$ E2 and $\mathrm{E} 1<<\mathrm{E} 2$.

In the period of E1>E2 and E1 $\leqslant \mathrm{E} 2$, the cluster is continuously embedded in the water film. For a small standoff distance, the stress region will first decrease clearly and then increase slowly again because of the occurrence of the split of the stress wave. Meanwhile, the high-pressure region of substrate will increase further. For a large standoff distance, both the stress region and high-pressure region will increase clearly again. However, at the end of E1 $\leqslant \mathrm{E} 2$ period, the stress region and the high-pressure region at large distances are still significantly less than that at small distances.

In the period of $\mathrm{E} 1<<\mathrm{E} 2$, the cluster cannot be embedded in the solidified water film. In addition, CN5 atoms begin to appear and increase rapidly until its maximum. In this period, both the stress region and high-pressure region do not change substantially, as the input energy is consumed by the generation of the substrate CN5.

There was no significant difference in the duration of $\mathrm{E} 1<<\mathrm{E} 2$ period between different distances. In addition, there is no obvious difference in the local pressure and temperature maximum of the impact zone between different distances at the moment of CN5max.

Therefore, it is the differences in the stress region and the high-pressure region that result in the reduction of the CN5 production rate at increasing distances. This eventually causes the CN5max of the small distance significantly greater than that at large distances.

\section{Conclusions}

In summary, the process of an abrasive water jet impinging on monocrystalline silicon substrate under different standoff distances was studied by molecular dynamics simulation. The influence of standoff distance on the damage of the crystal silicon substrate was 
studied by analyzing the variation of the CN5 atoms of the silicon substrate. The impact process before the CN5 atoms reach their maximum number is divided into three stages: solidification formation of water film, complete solidification of water film, and maintaining complete solidification of water film.

During the solidification formation stage, due to jet divergence, the solidified water film is relatively "thinner" and "denser" under the smaller standoff distance, and the forming time is short. On the contrary, under the larger standoff distance, the solidified water film is relatively "thicker" and "looser" with a longer formation time. Therefore, the stress region under the smaller distance is less than that of the larger distance. However, the high-pressure region of the former is larger than that of the latter.

At the stage of complete solidification, the "thicker" and "looser" water film will consume more input energy of the cluster to realize complete solidification. Therefore, the high-pressure region of small standoff distance continues to be larger than that of the large standoff distance, although both high-pressure regions are reduced at this stage. More importantly, the stress region of the former also begins to be significantly larger than the latter.

In the stage where complete solidification is maintained, the high-pressure region and the stress region of small distance are still far larger than those of large distance. These differences finally lead to CN5max and the damage degree of the substrate will first exhibit a small change and then decrease quickly with the increase in standoff distance. This phenomenon is consistent with the existence of an optimal standoff distance in the AWJ experiment.

In a word, at a nano-scale, jet divergence will affect the process of solidification of the water film between the cluster and the substrate under different standoff distances. This will change the energy transfer efficiency from the input jet to the impacted substrate. Therefore, the occurrence and maintenance of the complete solidification plays a decisive role in the damage formation of the silicon substrate. These findings are expected to be helpful for thoroughly understanding the material removal mechanism during AWJ or MJP process.

\section{Acknowledgements}

The work was financially supported by the National Natural Science Foundation of China (Nos. 51375291 and 91323302); Initial Research Funds for Young Teachers of Donghua University (No. 103-07-0053016); Innovation Program of Shanghai Municipal Education Commission (No. 13YZ004). The authors would also like to thank Prof. Hong Lei at Shanghai University and Prof. Xinchun Lu at Tsinghua University for their generous supports.

Open Access: The articles published in this journal are distributed under the terms of the Creative Commons Attribution 4.0 International License (http:// creativecommons.org/licenses/by/4.0/), which permits unrestricted use, distribution, and reproduction in any medium, provided you give appropriate credit to the original author(s) and the source, provide a link to the Creative Commons license, and indicate if changes were made.

\section{References}

[1] Hashish M. Visualization of the abrasive-waterjet cutting process. Exp Mech 28(2): 159-169 (1988)

[2] Guha A, Barron R M, Balachandar R. An experimental and numerical study of water jet cleaning process. J Mater Process Technol 211(4): 610-618 (2010)

[3] Fähnle O W, Brug H V, Frankena H J. Fluid jet polishing of optical surface. Appl Opt 37: 6771-6773 (1998)

[4] Horiuchi O, Ikeno J, Shibutani H, Suzuki H, Mizukami Y. Nano-abrasion machining of brittle materials and its application to corrective figuring. Precision Engineering 31: 47-54 (2007)

[5] Loc P H. Investigation of optimal air-driving fluid jet polishing parameters for the surface finish of N-BK7 optical glass. J Manuf Sci Eng 135(1): 228-229 (2013)

[6] Srinivasu D S, Axinte D A, Shipway P H, Folkes J. Influence of kinematic operating parameters on kerf geometry in abrasive waterjet machining of silicon carbide ceramics. Int J Mach Tool Manu 49(14): 1077-1088 (2009)

[7] Hashish M, Plessis M P D. Prediction equations relating high velocity jet cutting performance to stand off distance and multipasses. J Manuf Sci Eng 101(3): 311-318 (1979)

[8] Anglani F, Barry J, Dekkers W, Khare S. CFD modelling of a water-jet cleaning process for concentrated solar thermal 
(CST) systems. In Proceedings of third Southern African Solar Energy Conference, University of Pretoria, South Africa, 2015: 389-394

[9] He Z G, Li G S, Wang H Z. Shen Z G, Tian S C, Lu P Q, Guo B. Numerical simulation of the abrasive supercritical carbon dioxide jet: The flow field and the influencing factors. J Hydrodyn 28(2): 238-246 (2016)

[10] Kordonski W, Shorey A B. New magnetically assisted finishing method: material removal with magnetorheological fluid jet. Proc SPIE 5180: 107-114 (2004)

[11] Karakurt I, Aydin G, Aydiner K. An Experimental Study on the Depth of Cut of Granite in Abrasive Waterjet Cutting. Mater Manuf Process 27(5): 538-544 (2012)

[12] Zhang X C, Dai Y F, Li S Y, Peng X Q. Effect on material removal of magnetorheological jet polishing by several parameters. Opt Precision Eng 14(6): 1004-1008 (2006)

[13] Liu H, Wang J, Kelson N, Brown R J. A study of abrasive waterjet characteristics by CFD simulation. $J$ Mater Process Technol 153(1): 488-493 (2004)

[14] Momber A W, Kovacevic R. Principles of Abrasive Water Jet Machining. London (UK): Springer London, 1998

[15] Cleveland C L, Landman U. Dynamics of cluster-surface collisions. Science 257(5068): 355-361 (1992)

[16] Henkel M, Urbassek H M. Ta cluster bombardment of graphite: molecular dynamics study of penetration and damage. Nucl Instrum Methods Phys Res 145(4): 503-508 (1998)

[17] Insepov Z, Manory Z, Matsuo J, Yamada I. Proposal for a hardness measurement technique without indentor by gascluster-beam bombardment. Phys Rev B 61(13): 8744-8752 (2000)

[18] Yamaguchi Y, Gspann J. Large-scale molecular dynamics simulations of cluster impact and erosion processes on a diamond surface. Phys Rev B 66(15): 155408 (2002)

[19] Aokia T, Matsuo J. Surface structure dependence of impact processes of gas cluster ions. Nucl Instrum Methods Phys Res 216: 185-190 (2004)

[20] Han X, Gan Y X. Analysis the complex interaction among flexible nanoparticles and materials surface in the mechanical polishing process. Appl Surf Sci 257(8): 3363-3373 (2011)

[21] Chen R L, Liang M, Luo J B, Lei H, Hu X. Comparison of surface damage under the dry and wet impact: Molecular dynamics simulation. Appl Surf Sci 258(5): 1756-1761 (2011)

[22] Chen R L, Wu Y H, Lei H, Jiang R R, Liang M. Study of material removal processes of the crystal silicon substrate covered by an oxide film under a silica cluster impact: Molecular dynamics simulation. Appl Surf Sci 305(16): 609-616 (2014)

[23] Lv J, Bai M, Cui W, Li X. The molecular dynamic simulation on impact and friction characters of nanofluids with many nanoparticles system. Nanoscale Res Lett 6(1): 1-8 (2011)

[24] Aminfar H, Razmara N, Mohammadpourfard M. On flow characteristics of liquid-solid mixed-phase nanofluid inside nanochannels. J Appl Math Mech 35(12): 1541-1554 (2014)

[25] Algara-Siller G, Lehtinen O, Wang F C, Nair R R, Kaiser U, $\mathrm{Wu} \mathrm{H}$ A, Geim A K, Grigorieva I V. Square ice in graphene nanocapillaries. Nature 519(7544): 443-5 (2014)

[26] Bourg I C, Steefel C I. Molecular dynamics simulations of water structure and diffusion in silica nanopores. $J$ Phys Chem C 116(21): 11556-11564 (2012)

[27] Vollmayr K, Kob W, Binder K. Cooling-rate effects in amorphous silica: a computer- simulation study. Phys Rev B 54(22): 15808-15827 (1996)

[28] Rovere M, Ricci M A, Vellati D, Bruni F. A molecular dynamics simulation of water confined in a cylindrical SiO2 pore. J Chem Phys 108(23): 9859-9867 (1998)

[29] Watanabe T, Fujiwara H, Noguchi H, Hoshino T, Ohdomari I. Novel interatomic potential energy function for $\mathrm{Si}$, O mixed systems. Jpn J Appl Phys 38(38): 366-369 (1999)

[30] Jorgensen W J, Chandrasekhar J, Madura J D, Impey R W, Klein M L. Comparison of simple potential functions for simulating liquid water. J Chem Phys 79(2): 926-935 (1983)

[31] Tang C Y, Zhang L C. A molecular dynamics analysis of the mechanical effect of water on the deformation of silicon monocrystals subjected to nano-indentation. Nanotechnology 16(1): 15-20 (2005)

[32] Xu J, Luo J B, Lu X C, Wang L L, Pan G S, Wen S Z. Atomic scale deformation in the solid surface induced by nanoparticle impacts. Nanotechnology 16(6): 859-864 (2005)

[33] Evans D J, Hoover W G, Failor B H, Moran B, Ladd A J C. Nonequilibrium molecular dynamics via Gauss's principle of least constraint. Phys Rev A 28(2): 1016-1021 (1983)

[34] Chen R L, Luo J B, Guo D, Lei H. Dynamic phase transformation of crystalline silicon under the dry and wet impact studied by molecular dynamics simulation. $J$ Appl Phys 108(108): 073521 (2010)

[35] Hu J Z, Merkle L D, Menoni C S, Spain I L. Crystal data for high-pressure phases of silicon. Phys Rev B 34(7): 4679-4684 (1986) 


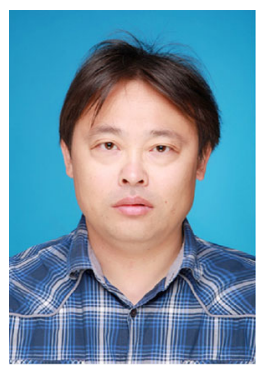

Ruling CHEN. He received his Ph.D. degree in mechanical engineering from the Tsinghua University in China in 2009. Now he is an associate professor at Donghua University. His research areas cover the nanotribology, ultra-precision surface machining/etc.

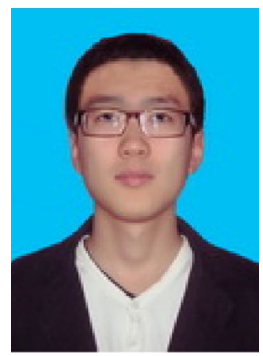

Di ZHANG. Master student in inorganic chemistry major at the Shanghai University. His research interest is molecular dynamics simulation and ultraprecision surface machining. 